\title{
Ontology based Knowledge Management for Administrative Processes of University
}

\author{
Anand Kumar \\ HLY Institute of Technology \& Management, Lucknow, 226002, India \\ E-mail: anand_smsvns@yahoo.co.in \\ Sanjay K. Dwivedi \\ Babasaheb Bhimrao Ambedkar University, Lucknow, 226025, India \\ E-mail: skd200@yahoo.com
}

\begin{abstract}
Knowledge management is a challenging task especially in administrative processes with a typical workflow such as higher educational institutions and Universities. We have proposed a system aSPOCMS (An Agent-based Semantic Web for Paperless Office Content Management System) that aims at providing paperless environment for the typical workflows of the universities, which requires ontology based knowledge management to manage the files and documents of various departments and sections of a university.

In Semantic Web, Ontology describes the concepts, relationships among the concepts and properties within their domain. It provides automatic inferring and interoperability between applications which is an appropriate vision for knowledge management. In this paper we discussed, how Semantic Web technology can be utilized in higher educational institution for knowledge representation of various resources and handling the task of administrative processes. This requires exploitation of knowledge of various resources such as department, school, section, file and employee etc. of the University by aSPOCMS which is built as an agent-based system using the ontology for communication between agent, user and for knowledge representation and management.
\end{abstract}

Index Terms-Ontology, Knowledge Management, aSPOCMS, Workflow.

\section{INTRODUCTION}

Knowledge Management is a big challenge for managing the knowledge and their representation especially in large organizations. Knowledge resides in mainly two extremes: (1) as explicit knowledge of documents and processes and (2) as inferred knowledge of people and procedures. When the organization needs to advance its knowledge management then it is important to focus on strategically important knowledge of the organization. It is also important to simplify that how the organization appreciates and defines the knowledge.

In the universe, the knowledge of human is not based only on facts which may be true or false. It is also based on certain and uncertain knowledge which is partially true or false. There are several methods which can be used to represent such knowledge for machine, e.g. probability measures, fuzzy logic or computing with word [1].
There are only strongly true facts and workflows in knowledge base of Universities. These facts and workflows are structured and defined by ontology. Ontology based knowledge is more simple and straight forward for agents to understand knowledge and also produces new knowledge from existing knowledge. The ontology based knowledge management of workflows and their resources improves administrative processes of the University in three ways [2]:

\section{A. Improving the Quality of Work}

Generally, the huge amounts of files or documents are processed to successfully accomplish the administrative work of the University. This kind of work requires welldefined knowledge of workflow. Agent can transmit the files and documents over well-defined workflow. The system based on ontology can encapsulate such knowledge to improve the quality of administrative work.

\section{B. Saving the Processing Cost}

System will provide the metadata of the resources (files or documents) and actions to be taken by previous user. This information can help to current user to take action at the resources. The electronic form of claim file will be transmitted through out the workflow of process. Therefore, this will be eliminated the printed papers and piles of files. The files or documents will be represented as URIs in the Semantic Web scenario. This will make the sharable of files or documents which will reduce the space to save the electronic files.

\section{Time Saving}

The files/documents can be rapidly and without any difficulty reused and processed according to ontological description within the system aSPOCMS (An Agentbased Semantic Web for Paperless office Content Management System) [3]. However, there is no need to manually find related file/document within system. Moreover, the system aSPOCMS will inform the users about the progress of current workflow activity and about the pending processes of the system $[4,5]$.

Ontology based knowledge management of departments, sections and workflow (of files and documents etc.) of a university in a precise and machine 
interpretable form for our system aSPOCMS is needed to process, share and reuse the knowledge of domain.

Therefore, to build the effective technologies for knowledge management, we need to know how the individuals or groups and organizations use the knowledge of the domain. In this sense, maximum knowledge of the domain should be represented in computer-readable forms which make the efficient use of knowledge to users as well as system. The computerreadable form of the knowledge can help to derive the inference of knowledge from existing knowledge. The main contribution of this paper is constructed the university ontology accordance of their resources by using a methodology.

\section{RELATED WORKS}

A number of research efforts are being made on ontology based knowledge management in various domains. It includes some projects that use ontologies with embedded knowledge for knowledge management system.

Kuo-Cheng $\mathrm{Ku}$ et al. [6] described a model of ontology-based knowledge management for joint venture projects. They defined a framework for an ontology based model to analyze the knowledge management processing of joint ventures in the Integrated Circuit industry. They have used a methodology to implement the ontology based knowledge management for joint venture projects with seven steps. These steps are involved in ontologybased knowledge management process model when implementing joint venture projects, which include knowledge acquisition, knowledge identification, ontology analysis, ontology implementation, ontology verification, knowledge reposition and knowledge sharing/development.

Laclavík M. et al. [7] presented the use of ontology for knowledge representation and handling for administrative processes. Using the CommonKADS methodology they have created the model of ontology based knowledge management for a public organization. It is further extended with the domain/application specific elements. They have taken the domain specific extension for the installation of Traffic Light in their representation and management of ontology based knowledge.

Budinska I., et al. [8] described a system architecture based on e-mail communication and a web portal suggested for ontology based knowledge management of workflow processes in administrative organizations. The ontology for the system has been designed from a detail analysis of workflow process associated with military training preparation and organization.

P. Sureephong, et al. [9] proposed an ontology based knowledge management system to support knowledge management activities within the industry cluster. They have described the methodology to capture, create and represent ontology for organization development by using the knowledge engineering approach. Furthermore, the handicraft cluster in Thailand is used as a case study to illustrate the proposed methodology.
Györgyi Elek, Bojana Dimić Surla et al. [10] are suggested the software architecture and implementation of the system for online public services based on the Semantic web technologies. They described the ontology for the certificate recognition service, software architecture and implementation that includes reading, processing and writing OWL, work with administrative documents and user interface.

Zhao Xiuxu, Zhu Yuming [11] focused on Application Research of Ontology-enabled Process FMEA Knowledge Management Method. They are designed the FMEA knowledge ontology by utilizing the visual modeling tool- Unified Modeling Language and constructs FMEA repository based on ontology. On the basis of FMEA repository, acquisition, storage and retrieval of FMEA knowledge during manufacturing process are achieved and requirements are satisfied for the manufacturing process quality continuous improvement via further application of FMEA knowledge ontology.

A. Macris, E. Papadimitriou and G. Vassilacopoulos [12] put the focus on ontology-based competency model for workflow activity assignment policies. In this context, they are developed an ontology-based competency model to assist in identifying the competencies that exist in an organization and the competencies required, by workflow activities and in performing a competency gap analysis as a prerequisite for domain-specific user development through competency-based training.

We observed form the review as presented in the previous sections that in order to manage the knowledge; ontology plays the significant role by enabling the content processing and sharing between experts and users. The ontological languages based on Web technology standards such as XML, RDF, RDFS and OWL are mainly used to implement the ontology. Thus, based on the reviews of various significant works, it deemed fit and quite reasonable to use a methodology for ontology design to represent and manage the knowledge of University for administrative process in paperless environment.

\section{ONTOLOGY AND KNOWLEDGE MANAGEMENT FOR ASPOCMS}

According to the Gruber [13] ontology is the "explicit specifications of a shared conceptualization". It is a conceptual model of facts in the world by identifying the relevant concepts of the phenomenon. The use of type of concepts and constraints are explicitly defined. Sharing of concepts means that ontology captures conceptual knowledge which is not private for individual, but accepted by the group. The role of ontology provides the facility to the construction of knowledge management processes of domain model. It provides a vocabulary of terms and relations in a specific domain. The ontology can be used to define the relationship among knowledge entities and return the knowledge to users [14] for their utilization. In building of a knowledge management of aSPOCMS, we need two types of knowledge [15] such as: 


\section{A. Domain Knowledge}

Domain Knowledge is the knowledge about a specific field of interest or subject. In ontology-based knowledge management for aSPOCMS, we need to represent domain knowledge about the objects of the University e.g. school, department, employee, workflow etc. Domain knowledge can be categorized in various level of ontology such as: top-level ontology, task ontology, domain ontology and application ontology. The ontology of domain knowledge can be used in several applications such that used as information systems for the domain. For example, ontology related to student in figure 1 can be used for student information system of University.

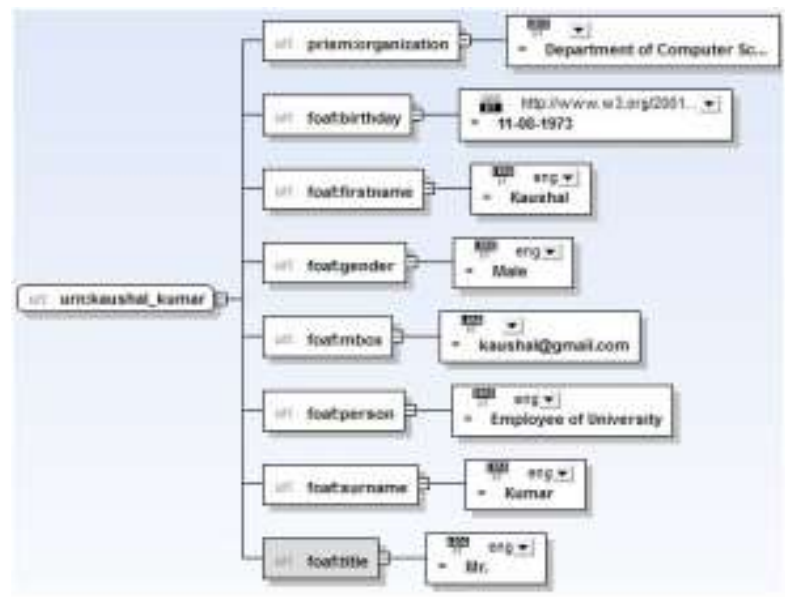

Fig. 1. Ontology of a Student's Information.

\section{B. Problem Solving Knowledge for Domain}

In the requirement of knowledge management of aSPOCMS, we must have the knowledge about how to use the domain knowledge to achieve the services of aSPOCMS in paperless environment. Problem solving knowledge has the ability to utilize previously learned knowledge in solving a new problem [16]. To develop the ontology of problem solving knowledge, it must identify major classes and instances of such knowledge of Universities that need to be communicated. The ontology related to university is represented in figure 2 with class, subclass and their instances.

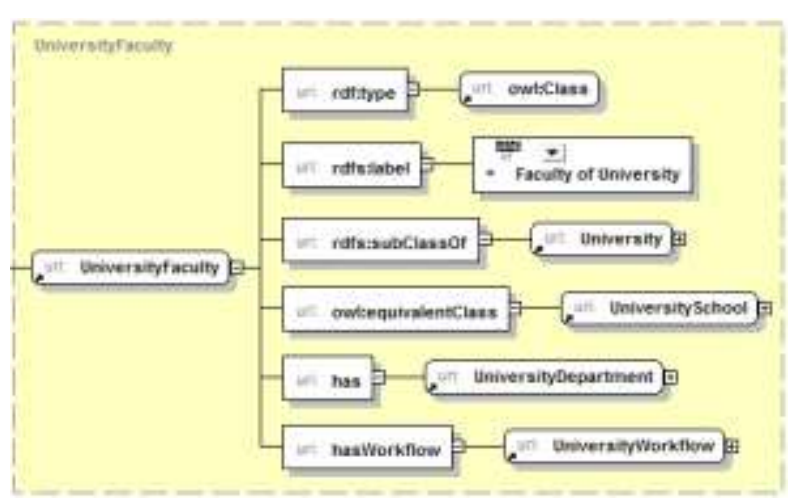

Fig. 2. Ontology with class, subclass and their instances.

In the figure, the resource UniversityFaculty is the equivalent class of UniversitySchool and subclass of University.

\section{ONTOLOGY BASED APPROACH FOR KNOWLEDGE MANAGEMENT}

The knowledge of higher educational institutions can classify as terms and concepts. The representation of terms and concepts has done with the help of ontology within computer science. Ontologies are useful because they encourage standardization of terms used to represent knowledge of a domain. However, ontology plays the crucial role in our system aSPOCMS, which can be used to derive the new knowledge from existing knowledge of discourse. Ontologies [17] are designed for being used in applications that need to process the content of information and reasoning about it, instead of just presenting information to humans. Ontologies permit machine for better interpretability of content than that supported by RDF [18, 19], RDFS [20, 21] formatted with XML [22]. The Ontology Web Language (OWL) [23, 24] for ontology design is providing additional vocabulary along with a formal semantics.

The paperless office content management system of University domain is defining and using ontologies of knowledge representation and their management. The ontology in the system is designed for the management of facts and workflow of University domain. The basic structure of knowledge of University can be represented by following figure 3:

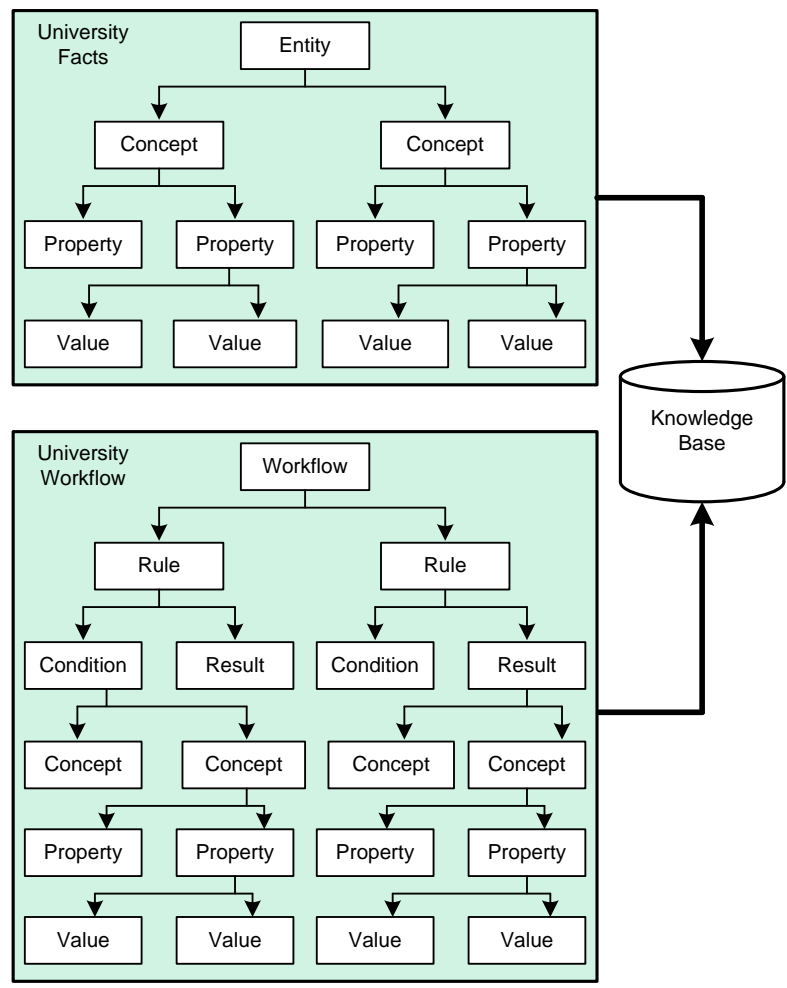

Fig. 3. General structure of Knowledge of the University.

The knowledge of University has two major parts: (1) facts of the University, (2) workflow of the University. Facts of the University are various entities or objects, which participate in various workflow of the University. Entity of the University has concepts, which will be represented by URI. The concepts have property with URIs or literal values. Workflow of the University has 
numbers of rules to execute the administrative activities of the University. Rules of the workflow may have some condition or produces the result. Further, the conditions or results can have some concept with property and their literal values.

\section{METHODOLOGY}

We have divided the ontology of universities into four levels: top-level ontology, domain ontology, application ontology and task ontology [25]. The explicit knowledge of University will represent in various level of ontology. In this work, we elaborate a methodology of ontologybased knowledge management for Universities. It involves various steps to represent the knowledge of universities. These steps are shown in figure 4:

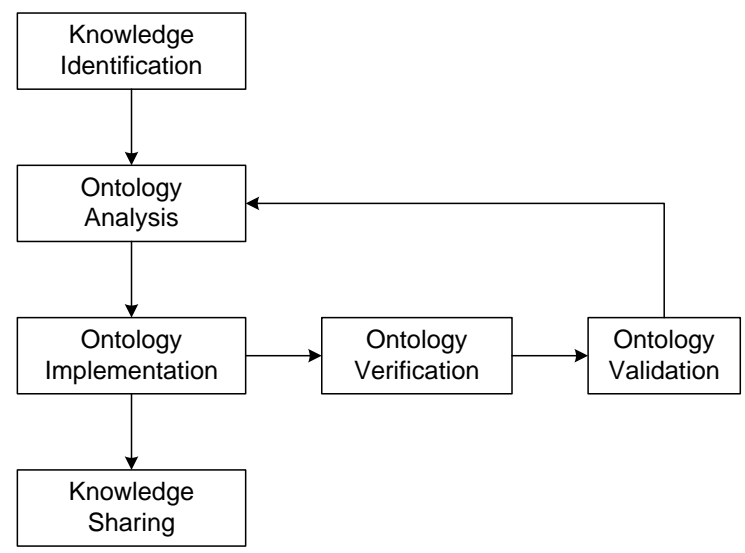

Fig. 4. A Methodology of Ontology-based Knowledge Management.

\section{A. Knowledge Identification}

Domain experts provide the explicit knowledge of the universities which can involve the overall knowledge of various resources and workflow processes. This knowledge is utilized by aSPOCMS services. The domain experts also identify that what knowledge needs to be shared and what knowledge does not need to be shared. Poor knowledge given by domain experts can lead to incorrect design of ontology and incomplete result of the system aSPOCMS. Therefore, it must be identify the explicit and correct knowledge of domain.

\section{B. Ontology Analysis}

Collected data and information of the universities are analyzed in this step. On the basis of analysis, the ontology of knowledge can be divided into various levels: top-level ontology, domain ontology, task ontology and application ontology. The ontology of university can be designed to achieve the expectation [26] of the Semantic Web technology in organization and the system aSPOCMS. The ontology analysis can also verify that how the ontology is useful to our system aSPOCMS. In this step, we will analyze the concept, individuals and conceptual roles of the universities.

\section{Ontology Implemantation}

The ontology implementation executes the process of ontology design and ensures that various levels of ontology of universities are designed in this step after the analysis of ontology. We will use the ontology development tools for ontology-based knowledge management [27].

\section{Ontolgoy Verification}

It includes the verification of various concepts such as:

- Definition and axiom of each individuals.

- Collections of definitions and axioms that are stated explicitly in the definitions of the ontology.

- Axioms that can be inferred using definitions [28].

The ontology verification also checks that the ontology satisfies particular constraints or not.

\section{E. Ontology Validation}

The consistency of ontology with respect to its applications is done in ontology validation. Validation of University ontology has be done by the defined concepts, axioms and assertions etc. of ontology and identified knowledge of University.

\section{F. Knowledge Sharing}

The resources of the universities consists the definitions, metadata and axioms for aSPOCMS. The resources represented by URIs and metadata of the resources can be represented by using namespaces, which makes the resources and their metadata shared among the universe.

\section{KNOWLEDGE MANAGEMENT REPOSITORY OF UNIVERSITIES FOR ASPOCMS}

The knowledge base of the system aSPOCMS is related to user's profile (Employees, Student and Visitor etc.), organizational structure and workflow of higher educational universities, which encapsulates the users and their activities. The representation of relevant knowledge can be based on primitive concepts from various level of ontology. Ontologies are designed for one or several purpose, which may be organized into libraries. However, these libraries are enhancing the reusability.

When ontology of a specific domain is large then the tools for their management must be used. The tools can help with ontology verification and validation. The verification of ontology checks whether ontology satisfies particular constraints or not. For example, cardinality constraints for entity-relationship can be checked by the verification tools and the consistency of model with respect to its application. To construct the University ontology, we have used the methodology for ontology based knowledge management, which is described in this paper previously. The framework of each steps of methodology for the construction of University ontology is as following:

\section{A. Identification of Knowledge of Universities}

The ontology must have explicit knowledge of various resources of universities. The ontology for aSPOCMS involves the information of organizational structure, profile of employee, student profile and various workflows of processes of universities as knowledge. The 
integrated framework of this knowledge is used for aSPOCMS services. Illustration of some information of typical organizational structure and workflow of University is as given below:

Information of organizational structure:

- University has various workflows.

- University has people of University.

- People of University are employee and student of University.

- University has schools.

- School of University is equivalent or same as faculty of University.

- School has various centre or department.

- University has various courses.

- University has administrative sections. Information of workflow:

- File from a department related to finance is forwarded to head of department.
- Head of department forwarded the file to Assistant Registrar (Finance).

Then, the file is forwarded to Finance Officer.

\section{B. Ontology Analysis of Information}

After the knowledge identification, we have analyzed the ontology classes (owl:Class) and object properties of ontology (owl:ObjectProperty) of identified knowledge. The classes have individuals, which is represented by URIs. Object properties are used to represent the relationship between classes. We have conceptualized the information of University by using OWL concepts such as owl:Class, owl:unionOf and owl:ObjectProperty etc. These concepts are used to construct ontology of identified knowledge. A typical example of OWL classes, object properties and individuals of universities is shown in tables 1, 2 and 3 respectively:

Table 1. Classes of University Ontology and their Definition

\begin{tabular}{|c|c|}
\hline OWL Classes & Definitions of Classes \\
\hline University & Class of University of Country of India \\
\hline UniversityWorkflow & Contains the various workflow of University \\
\hline UniversityPeople & Have the people of University \\
\hline UniversityEmployee & Class of employees of University \\
\hline UniversityStudent & Has all student of University which enrolled in various courses in University \\
\hline UniversitySchool & Contains the schools of University \\
\hline UniversityFaculty & Has the faculty of University \\
\hline SchoolCentre & Contains the centre of schools \\
\hline UniversityCources & Have the various courses of University \\
\hline AdministativeBlock & Has all administrative section of administrative block \\
\hline
\end{tabular}

Table 2. Some Object Properties and their Definitions

\begin{tabular}{|c|c|}
\hline Object Properties & Definition of Object Properties \\
\hline unionOf & Super class has subclasses by using owl:unionOf property. \\
\hline equivalentClass & Relationship among similar classes are represented by owl:equivalentClass. \\
\hline has & Individual of a class has individual of other class. \\
\hline sameClass & One class is same as other class. \\
\hline
\end{tabular}

Table 3. List of Individuals their respective OWL Classes and Definition

\begin{tabular}{|c|c|c|}
\hline Individuals & OWL Classes & Definition of Individuals \\
\hline InternationalStudies & UniversitySchool & School of International Studies \\
\hline LLCStudies & UniversitySchool & School of Language, Literature and Culture Studies \\
\hline SocialSciences & UniversitySchool & School of Social Sciences \\
\hline Arts & UniversityFaculty & Faculty of Arts \\
\hline CommerceBusinessStudies & UniversityFaculty & Faculty of Commerce \& Business Studies \\
\hline Education & UniversityFaculty & Faculty of Education \\
\hline EuropeanStudies & SchoolCentre & Centre for European Studies \\
\hline EastAsianStudies & SchoolCentre & Centre for East Asian Studies \\
\hline Linguistics & SchoolCentre & Centre for Linguistics \\
\hline PoliticalStudies & SchoolCentre & Centre for Political Studies \\
\hline HumanRights & UniversityDepartment & Department of Human Rights \\
\hline HMLanguage & UniversityDepartment & Department of Hindi and Modern Language \\
\hline
\end{tabular}




\section{Implementation of Ontology of Knowledge of Universities}

University Ontology consists of hierarchical description of concepts such as super class, sub class and descriptions of the properties of each class. According to analyzed concepts of knowledge in table 1, 2 and 3 in previous subsection, we construct the ontology of identified knowledge by using ontology editor tool. There are many ontology editor tools such as Portege, Neon, Altova Semantic Works, Pool Party and Neologism etc. We have used SemanticWorks 2009 [29] to implement the ontological concepts of the information. The graphical view of ontology based information and analyzed concepts of identified knowledge of University are represented in figure 5 and a part of OWL code is illustrated in figure 6.

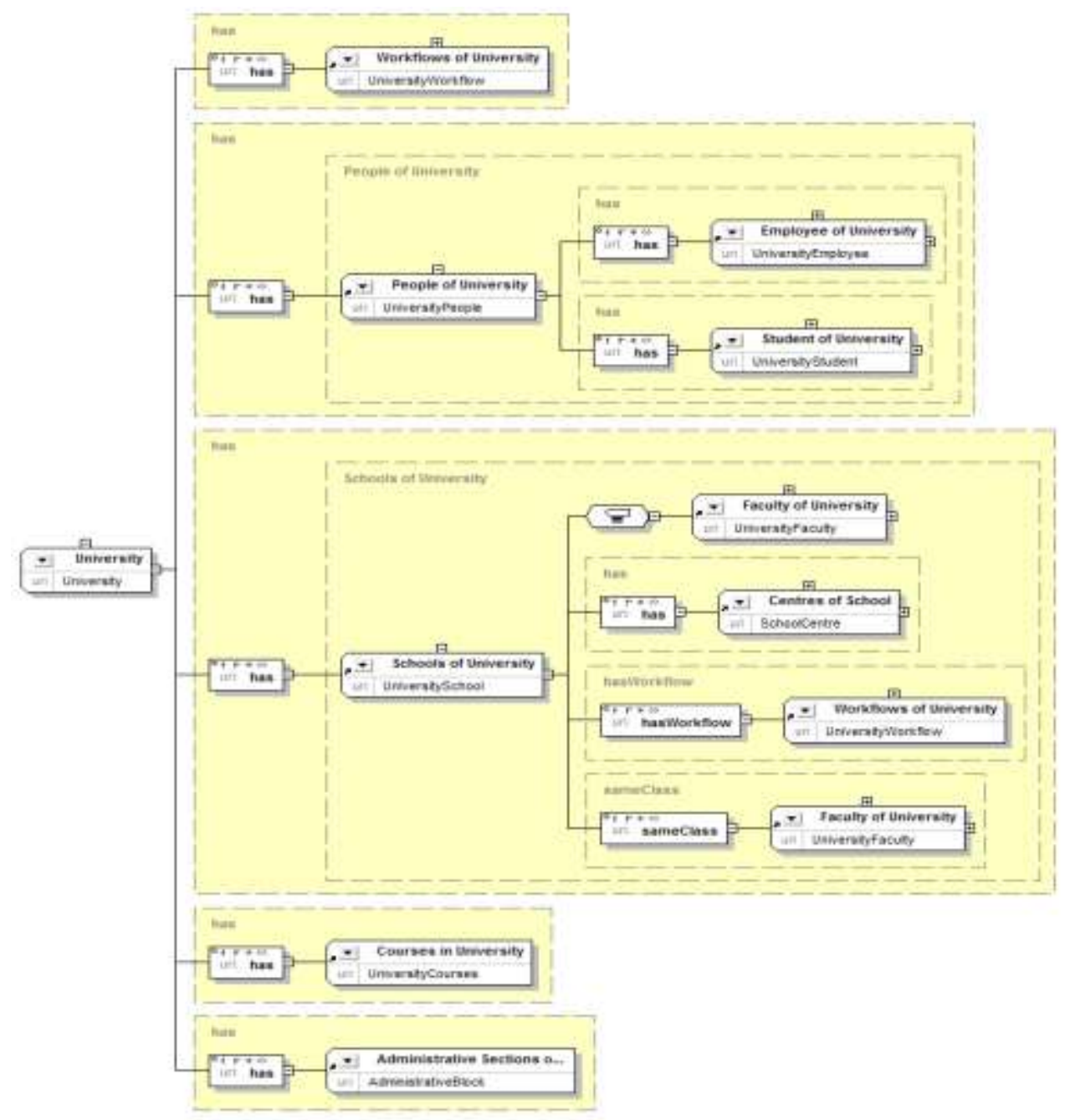

Fig. 5. Graphical representation of University Ontology by using SemanticWorks.

$<$ ?xml version="1.0"?>

<rdf:RDF xml:base="http://www.xyzu.ac.in/univont" xmlns="http://www.xyzu.ac.in/univ-ont\#" xmlns: owl="http://www.w3.org/2002/07/owl\#" xmlns:uniwf="http://localhost/ontology/uniwf.o wl\#">

$<$ rdf: Description

rdf:about="\#UniversityWrokflow">

$<$ rdf:type>

$<$ rdf:Description

rdf:about="http://www.w3.org/2002/07/owl\#Class

$" />$

$</$ rdf : type $>$

<rdfs:label>Workflow of

University</rdfs: label>

<rdfs:subClassof>

<rdf:Description rdf:about="\#University"/>

$</$ rdfs: subclassof $>$

$</$ rdf: Description $>$

Fig. 6. Part of OWL code of University Ontology.

\section{Verification of University Ontology}

As discussed in previous subsection, now we verify the University ontology at local host. We used RacerPro Version 2.0 [30] inference engine to verify the various concept, assertions and axioms etc. of University ontology. It verifies the concepts, roles, individuals, assertions, axioms, taxonomy and role hierarchy etc. which is defined in ontology on the basis of knowledge. University ontology verification shows that definition and axioms of each individuals of university are accurate. Figure 7 represents the axioms in University ontology, which show that the concepts of identified information of university are well defined in ontology. 


\begin{tabular}{|lll}
\hline ID & Axiom Type \& Attribute Type & Avguments \\
\hdashline 24 & ObjectPropertyAssertionAxiom & LOADED \\
\hline 24 & SUBJECT & \#:University \\
24 & REL-OBJ.............. \\
24 & OBJECT & \#:has \\
& & \#:UniversityWorkflow \\
25 & ObjectPropertyAssertionAxiom & LOADED \\
25 & SUBJECT & \#:University \\
25 & REL-OBJECT-PROPERTY & \#:has \\
25 & OBJECT & \#:UniversityPeople \\
& & \\
26 & ObjectPropertyAssertionAxiom & LOADED \\
26 & SUBJECT & \#:University \\
26 & REL-OBJECT-PROPERTY & \#:has \\
26 & OBJECT & \#:UniversitySchool \\
& & \\
27 & ObjectPropertyAssertionAxiom & LOADED \\
27 & SUBJECT & \#:University \\
27 & REL-OBJECT-PROPERTY & \#:has \\
27 & OBJECT & \#:UniversityCourses \\
& & \\
28 & ObjectPropertyAssertionAxiom & LOADED \\
28 & SUBJECT & \#:University \\
28 & REL-OBJECT-PROPERTY & \#:has \\
28 & OBJECT & \#:AdministrativeBlock \\
& &
\end{tabular}

Fig. 7. Axioms in University Ontology by using RacerPro.

\section{E. Validation of University Ontology}

The validation of University ontology has been done manually on the basis of ontology verification and knowledge identification. We verified the ontology of University by using the inference engine as discussed in previous subsection. In this section, we validated the concepts, individuals and role of the concepts, assertions, axioms, taxonomy and hierarchy of the role defined in University Ontology. The assertions of ontology validate the information, which is represented in University ontology. Some of the assertions of University ontology are shown in figure 8 .

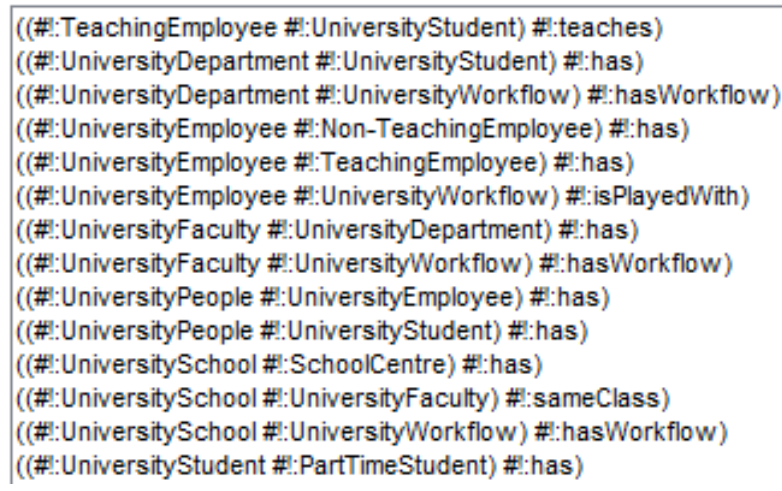

Fig. 8. Snapshot of Assertions of Ontology identified by RacerPro 2.0.

In figure $\quad 8, \quad$ statement ((\#!:TeachningEmployee\#!:UniversityStudent) \#!:teaches) is representing that property 'teaches' is established relationship between TeachingEmployee and UniversityStudent. Wherein, TeachingEmployee and UniversityStudent are the OWL classes. Similarly, some information from the statements of figure 7 is shown below, which identified in subsection 6.1:
- Teaching employee teaches the University student.

- Department has the University student.

- Department has University workflow.

- University employee has non-teaching employee.

- University employee is played with University workflow.

Some information is conceptualized as axioms (subject, relational object-property and object) in ontology representation and ontology based knowledge management. Some axioms are identified by inference engine RacerPro 2.0 as in figure 9, which conceptualized in ontology representation of information. These axioms are represented in ontology on the basis of table 1, 2 and 3 , which is discussed in ontology analysis sub section.

\begin{tabular}{|c|c|c|}
\hline 10 & Axiom Type \& Attribute Type & Avguments \\
\hline 24 & ObjectProperty AssertionAxiom & LOADED \\
\hline 24 & SUBJECT & A: University \\
\hline 24 & REL-OBJECT-PROPERTY & \#:has \\
\hline 24 & OBJECT & \#:UniversityWorkflow \\
\hline 25 & ObjectPropertyAssertionAxiom & LOADED \\
\hline 25 & SUBJECT & \#:University \\
\hline 25 & REL-OBJECT-PROPERTY & Whas \\
\hline 25 & OBJECT & \#:UniversityPeople \\
\hline 26 & ObjectPropertyAssertionAxiom I & LOADED \\
\hline 26 & SUBJECT & \#:University \\
\hline 26 & REL-OBJECT-PROPERTY & \#:has \\
\hline 26 & OBJECT & W. Universityschool \\
\hline 27 & ObjectPropertyAssertionAxiom & LOADED \\
\hline 27 & SUEUECT & w: University \\
\hline 27 & REL-OBJECT-PROPERTY & \#:has \\
\hline 27 & OBJECT & 4.:Universitycourses \\
\hline 28 & ObjectPropertyAssertionAxiom & LOADED \\
\hline 28 & SUEJECT & 4. University \\
\hline 28 & REL-OBJECT-PROPERTY & W:has \\
\hline 28 & OBJECT & \#:AdministrativeElock \\
\hline 32 & SubClassAxiom & LOADED \\
\hline 32 & SUE-CLASS & H: Schooicentre \\
\hline 32 & SUPER-CLASS & \#:Universityschool \\
\hline 33 & SubClassAxiom & LOADED \\
\hline 33 & SUB-CLASS & \#:SchoolCentre \\
\hline 33 & SUPER-CLASS & \#:University Faculty \\
\hline 34 & EquivalentClasses Axiom & LOADED \\
\hline 34 & DESCRIPTIONS & (\#:SchoolCentre \#:UniversityDepartment) \\
\hline 37 & SubClassaxiom & LOADED \\
\hline 37 & SUB-CLASS & \#:UniversityDepartment \\
\hline 37 & SUPER-CLASS & W: Universityf aculty \\
\hline 38 & SubCiasaAxiom & LOADED \\
\hline 38 & SUE-CLASS & w:UniveraityDepartment \\
\hline 38 & SUPER-CLASS & \#:Universityschool \\
\hline 39 & EquivalentClassesaxiom & LOADED \\
\hline 39 & DESCRIPTIONS & (*f:UniversityDepartment $\#$ : Schooicentre) \\
\hline 40 & ObjectPropertyAssertionAxiom & LOAOEO \\
\hline 40 & SUBJECT & \#:UniversityDepartment \\
\hline 40 & REL-OBJECT-PROPERTY & Whas \\
\hline 40 & OBNECT & \#:Universitystudent \\
\hline 41 & ObjectPropertyAssertionAxiom & LOADED \\
\hline 41 & SUEJECT & \#:UniversityDepartment \\
\hline 41 & REL-OBJECT-PROPERTY & \#:hasWorkfiow \\
\hline 41 & OBJECT & W. UniversityWorkflow \\
\hline 44 & EquivalentClassesaxiom & LOADED \\
\hline 44 & DESCRIPTIONS & (w:UniversitySchool w: Universityf aculty) \\
\hline
\end{tabular}

Fig. 9. Axiom Type \& Attribute Type and their Arguments of defined information in ontology.

In figure 9, illustration of axiom id: 24 has the subject, object-property and object as 'University', 'has' and 'UniversityWorkflow' respectively. Therefore, the information of axiom id: 24 are 'University has University Workflow'. Similarly, the information of some other axioms id of figure 7 is as following table:

In order to check the taxonomy constructed in university ontology, we used a set of queries are fetched via inference engine RacerPro 2.0. The results of three sample set of queries are shown in table 5: 
Table 4. Information from Axioms of University Ontology

\begin{tabular}{|c|c|}
\hline Axioms id & Information from axioms id \\
\hline 25 & University has people of university. \\
\hline 26 & University has schools of University. \\
\hline 27 & University has University courses. \\
\hline 28 & University has administrative block. \\
\hline 32 & Centre of school is the subclass of university school. \\
\hline
\end{tabular}

Table 5. Query and Result of University Ontology

\begin{tabular}{|l|c|}
\hline \multicolumn{1}{|c|}{ Query } & Result \\
\hline ? (atomic-concept-synonyms & \\
\#!:UniversityDepartment & (\#!:UniversityDepartment \\
file://E:/University\%20Ontolo & \#!:SchoolCentre) \\
gy/uni-onto.owl) & \\
\hline ? (atomic-concept-synonyms & \\
\#!:UniversitySchool & (\#!:UniversityFaculty \\
file://E:/University\%20Ontolo & \#!:UniversitySchool) \\
gy/uni-onto.owl) & \\
\hline ?(retrieve & \\
(?X) & \\
(?X \#!:UniversityPeople) & \\
:abox & \\
file://E:/University\%20Ontolo & NIL \\
gy/uni-onto.owl) & \\
\hline
\end{tabular}

First two queries have returned the synonyms of OWL class UniversityDepartment and UniversitySchool as SchoolCentre and UniversityFaculty respectively. The third query is retrieved the individuals of OWL class UniversityPeople, which is NIL. The OWL class UniversityPeople has no individuals in our constructed university ontology. By using queries and their result, here we have verified the taxonomy constructed in university ontology.

\section{F. Sharing of Knowledge of University}

The sharing and reuse of knowledge of ontology does not appear only at the data level, but also at the application level in Semantic Web. Nowadays, there are many existing ontology from a wide range of sources that can be reused when building Semantic Web Applications. Ontologies provide the shared and reused knowledge and ontology across the applications. After the verification and validation, the concepts and individuals etc. of the university defined in ontology is ensured to correct. As a final point, university ontology is ready to use for the system aSPOCMS and other applications.

The resources of the information of university are represented as URIs. The URI makes the global sharing of knowledge among the universe. The content defined by URIs is easily understandable and processible by computer. Any user can access the content of ontology by using their URIs. Some part of axioms with URIs of University ontology (uni-onto) is depicted as follows:

In axioms of university ontology, URI http://www.xyzu.ac.in/univ-ont\#Notice is stand for a notice of university, which is access by any user with their URI. Similarly, http://www.xyzu.ac.in/univont\#Examination stands for a semester examination of university. Therefore, this ontology is used as library of university ontology, which can be shared by using their URI.

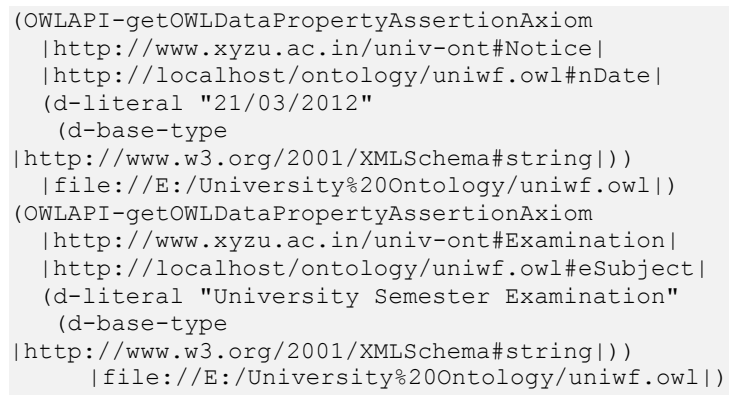

Fig. 10. Some axioms of university ontology identified by RacerPro 2.0.

\section{DISCUSSION}

Various literatures on ontology based knowledge management consent that ontology in knowledge management is used to enable the machine with content readable and processible and enhance the knowledge sharing, reusing and querying on knowledgebase to retrieve the result in accordance with taxonomy constructed in university ontology. Various methodologies are used to implement the ontology based knowledge management in different domains. For instance, knowledge of joint venture projects [6] represented the ontology by using a methodology which provides a knowledgebase for joint venture projects. The business processes and related information of joint venture projects are involved in their ontological knowledgebase.

With the requirement of our system aSPOCMS, knowledge of well defined administrative processes (workflows) of University is represented as ontology. The university ontology has the knowledge of employees with their authorization, knowledge of various departments with their hierarchical relationship (such as super class and subclass etc.) and the knowledge regarding well defined workflow and information of documents/files associated with workflows. In some citations, researchers have used ontology for various works related to university. For example, Ling Zeng, et al. 2009 [31] introduced University ontology for teaching purpose based on University courses, in which authors have described the information of courses, content of courses etc.

In our ontological approach, we introduced a methodology to design the ontology based knowledge management, in which OWL is used to conceptualize the knowledge of various resources of University. As an advancement of University ontology, we extended the ontology with extra knowledge and features such as knowledge of employees, workflow and documents/files etc. with access control (authorization). The documents/files navigate over well defined workflows (various departments for administrative process) designed in University ontology based on knowledge (metadata) of employees, documents, departments and workflows. The DL-OWL ontology language and ontology editing tools have been used to construct the university ontology [32] and verified the logical concepts, axioms and taxonomy of constructed ontology by using RacerPro inference 
engine. Furthermore, ontology query language is used to verify the defined taxonomy in university ontology.

\section{CONCLUSION}

The development of ontology for knowledge management depicts the conceptualization to be modelled and the vocabulary with its axioms to give the meaning of application domain of higher educational institutions such as University. Vocabulary and its axioms set up the logical design of a mechanism to manage the ontology. A methodology has been introduced to construct the ontology based knowledge management of university for our system aSPOCMS. The methodology to construct the university ontology uses a suitable ontology language, ontology editing tools and inference engine to verify the concepts, individuals, their roles, assertions, axioms and taxonomy and role hierarchy of the ontology. The taxonomy has verified by using ontology query language. The verification of ontology has been done on account of defined concepts in ontology and identified knowledge.

\section{REFERENCES}

[1] Paul P. Wang (Editor): Computing with Words, ISBN: 0471-35374-4, (2001).

[2] Ted Williams. "Workflow Management within the ARIS Framework",

http://www.pera.net/Methodologies/ARIS/ARIS_Paper_by _Ted_Williams.html.

[3] Sanjay K. Dwivedi and Anand Kumar, "An Agent-based Semantic Web for Paperless Office Content Management System", ICDM 2010: Proceedings of the 2010 Third International Conference on Data management, pp. 352360, Ghaziabad, India, March 2010.

[4] Blum, B.L. (1989, October). Toward a paperless development environment. Proceedings of the IEEE International Workshop on Architectures, Languages and Algorithms (pp. 495-498), Fairfax, VA.

[5] Breaux, P.J. (1994, September). "Using computer technology to support cost-effective paperless interactive technical data". Proceedings of the IEEE Systems Readiness Technology Conference on Cost Effective Support into the Next Century (pp. 83-87), Anaheim, CA.

[6] K. C. Ku, A. Wensley, H. P. Kao (2008), "Ontology based knowledge management for joint venture projects", Expert Systems With Applications, Vol. 35, Issue 1-2, July, 2008 (pp. 187-197) USA.

[7] Laclavík M., Z. Balogh, L. Hluchy, K. Krawczyk, M. Dziewierz, J. Kitowski, M. Majewska, "Knowledge Management for Administration Processes", Proc. of Znalosti 2004, February 2004, pp. 248-255. ISBN 80-2480456-5.

[8] Budinská I., Gatial E., Nguyen G., Laclavík M., Balogh Z., Forgác R., Mokriš I., Hluchý L., Ciglan M., Babík M., "Ontology based Knowledge Management for Organizational Learning”, http://ftp.informatik.rwthaachen.de/Publications/CEUR-WS/Vol-180/paper19.pdf.

[9] P. Sureephong, N. Chakpitak, Y. Ouzrout, A. Bouras, “An Ontology based Knowledge Management System for Industry Clusters", International Conference on Advanced Design and Manufacture proceeding, January 2008.
[10] Györgyi Elek, Bojana Dimić Surla, Zora Konjović, "Implementation of the ontology-based Online Public Service", Proceedings of the $6^{\text {th }}$ PSU-UNS International Conference on Engineering and Technology (ICET-2013), Novi Sad, Serbia, May 15-17, 2013, pp. 1-4.

[11] Zhao Xiuxu, Zhu Yuming, "Application Research of Ontology-enabled Process FMEA Knowledge Management Method", International Journal of Intelligent Systems and Applications, MECS Press USA, Vol. 3, April 2012, pp. 34-40.

[12] A. Macris, E. Papadimitriou and G. Vassilacopoulos, "An ontology-based competency model for workflow activity assignment policies", Journal of Knowledge Management, VOL. 12 NO. 62008 , pp. 72-88.

[13] Gruber TR, (1991) "The Role of Common Ontology in Achieving Sharable, Reusable Knowledge Bases”, In J. A. Allen, R. Fikes, \& E. Sandewall (Eds.), Principles of Knowledge Representation and Reasoning: Proceedings of the Second International Conference, Cambridge, MA, pp. 601-602.

[14] Anand Kumar, Sanjay K. Dwivedi (2011). "Semantic Web Expectations and Challenges in aSPOCMS", International Journal on Recent Trends in Engineering \& Technology [IJRTET], Volume 5, Issue 1, March 2011, pp. 60-65.

[15] Youn, Seongwook and McLeod, Dennis, "Ontology Development Tools for Ontology-Based Knowledge Management" (2006). Non-published Research Reports, Paper 100.

[16] Go'mez-Pe'rez, A. (1996). "Towards a framework to verify knowledge sharing technology. Expert System with Applications", 11(4), 519-529.

[17] V. Aida, G. Karina, S. David, B. Montserrat, (2010), "Using ontologies for structuring organizational knowledge in Home Care assistance". International Journal of Medical Informatics $\left.79 \begin{array}{lllll}2 & 0 & 1 & 0\end{array}\right)$ 370-387, doi:10.1016/j.ijmedinf.2010.01.012,Elsevier Ireland Ltd.

[18] O. Lassila and R. R. Swick (editors). "Resource description framework (rdf) model and syntax specification". http://www.w3.org/TR/1999/REC-rdf-syntax-19990222/, Feb, 1999.

[19] W3C, "RDF Primer", W3C Recommendation, Retrieved from, http://www.w3.org/TR/2004/REC-rdf-primer, February 2004.

[20] Brickley, D. \& Guha, R. eds. (2002), "Resource description framework (RDF) schema specification". W3C Working Draft 30 April 2002 Cambridge, MA: W3C.

[21] Resource Description Framework (RDF) Schema Specification 1.0, retrieved October 10, 2004 from http:/www.w3.org/TR/rdf-schema.

[22] XML (2003). XML: extensible Markup Language, retrieved October 10, 2004 from http://www.w3.org/XML/.

[23] OWL Web Ontology Language Reference. http://www.w3.org/TR/owl-ref/.

[24] Fensel, D., van Harmelen, F., Horrocks, I., McGuinness, D L., \& Patel-Schneider, P. F. (2001). "OIL: An ontology infrastructure for the semantic web". IEEE Intelligent Systems, 16(2):38-44.

[25] M. Laclavik, Z. Balogh, L. Hluchy, R. Słota, M. Dziewierz, K Krawczyk, "Distributed Knowledge Management based on Software Agents and Ontology". PPAM Conference (2003).

[26] Chandrasekaran B, Josephson, JR, Richard BV, (1998), "Ontology of Tasks and Methods", In Workshop on Knowledge Acquisition, Modeling and Management (KAW'98), Canada.

[27] Mayer, R., \& Wittrock, M., (1996), "Problem solving and transfer", In D. Berliner, \& R. Calfee (Eds.), Handbook of 
educational psychology (pages 45-61). New York: Macmillan.

[28] Kumar, A., Dwivedi S. K. "Ontology Exemplification for aSPOCMS in the Semantic Web", World Congress on Information and Communication Technologies (WICT) 2011. IEEE Xplore, pp. 473-478.

[29] SemanticWorks Semantic Web tool - Visual RDF and OWL editor, http://www.altova.com/semanticworks.html.

[30] RacerPro Version 2.0, Retrieved from, http://www.racersystems.com/products/download/index.ph tml.

[31] Ling Zeng et al. - Study on Construction of University Course Ontology: Content, Method and Process IEEE2009.

[32] Sanjay K. Dwivedi and Anand Kumar, "Development of University Ontology for aSPOCMS", Journal of Emerging Technologies in Web Intelligence, Academy Publisher, Vol. 5, No. 3, August 2013, pp. 213-221.

\section{Authors' Profiles}

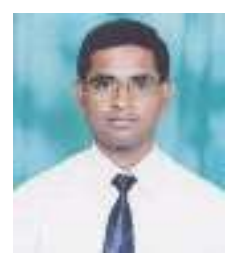

Anand Kumar, Assistant Professor in Department of Computer Science and Engineering at HLY Institute of Technology and Management, Lucknow - 226002 (U.P.) India. His research interest is in Semantic Web and Ontology design. $\mathrm{He}$ is approachable at anand_smsvns@yahoo.co.in.

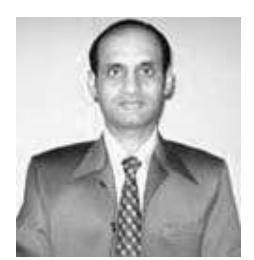

Sanjay K. Dwivedi, Associate Professor \& Head, Department of computer science at Babasaheb Bhimrao Ambedkar University, Lucknow -226025 (U.P.) India (A Central University). His research interest is in Artificial Intelligence, Data Mining, Search Engines and e-Governance. He has published number of research papers in journals and conferences both. He is approachable at skd200@yahoo.com.

How to cite this paper: Anand Kumar, Sanjay K. Dwivedi,"Ontology based Knowledge Management for Administrative Processes of University", International Journal of Information Technology and Computer Science(IJITCS), vol.7, no.8, pp.51-60, 2015. DOI: 10.5815/ijitcs.2015.08.08 